\title{
Time dependence of the velocity autocorrelation function of a fluid: An eigenmode analysis of dynamical processes
}

\author{
S. Bellissima, ${ }^{1}$ M. Neumann, ${ }^{2}$ E. Guarini, ${ }^{1}$ U. Bafile,,${ }^{3, *}$ and F. Barocchi ${ }^{1}$ \\ ${ }^{1}$ Dipartimento di Fisica e Astronomia, Università degli Studi di Firenze, via G. Sansone 1, I-50019 Sesto Fiorentino, Italy \\ ${ }^{2}$ Fakultät für Physik der Universität Wien, Strudlhofgasse 4, A-1090 Wien, Austria \\ ${ }^{3}$ Consiglio Nazionale delle Ricerche, Istituto dei Sistemi Complessi, via Madonna del Piano 10, I-50019 Sesto Fiorentino, Italy
}

(Received 6 June 2015; published 30 October 2015)

\begin{abstract}
The velocity autocorrelation function (VAF), a key quantity in the atomic-scale dynamics of fluids, has been the first paradigmatic example of a long-time tail phenomenon, and much work has been devoted to detecting such long-lasting correlations and understanding their nature. There is, however, much more to the VAF than simply the evidence of this long-time dynamics. A unified description of the VAF from very short to long times, and of the way it changes with varying density, is still missing. Here we show that an approach based on very general principles makes such a study possible and opens the way to a detailed quantitative characterization of the dynamical processes involved at all time scales. From the analysis of molecular dynamics simulations for a slightly supercritical Lennard-Jones fluid at various densities, we are able to evidence the presence of distinct fast and slow decay channels for the velocity correlation on the time scale set by the collision rate. The density evolution of these decay processes is also highlighted. The method presented here is very general, and its application to the VAF can be considered as an important example.
\end{abstract}

DOI: 10.1103/PhysRevE.92.042166

PACS number(s): 61.20.Lc, 05.20.Jj, 05.10.-a

\section{INTRODUCTION}

The translational dynamics of fluids is mainly characterized by the two phenomena of diffusion and vibrational motions, either localized or propagating in form of acoustic waves [1,2]. Besides self (i.e., single-particle) processes like mass diffusion, also sound propagation, transverse dynamics, and other collective phenomena have clear reflections onto the motions of individual particles $[3,4]$. Thus, a simply defined self quantity like the velocity autocorrelation function (VAF) reflects more or less directly the whole variety of fluid dynamics, which originates at a microscopic scale but shows a rather direct connection to macroscopic, measurable quantities, such as the diffusion coefficient, and the sound speed and damping. This fact provided good reasons for studying the VAF, mainly by means of theoretical or simulation methods $[5,6]$. However, the most important reason has been for a long time a phenomenon evidenced by the surprising findings of Alder and Wainwright [7,8].

These authors detected, in a molecular dynamics (MD) simulation of a hard-sphere (HS) fluid at intermediate density, a long-time VAF dependence displaying a characteristic slow decay, well represented by the power law behavior $t^{-3 / 2}$. This unexpected result was the first appearance of a so-called long-time tail (LTT) in the microscopic dynamics of fluids. The effect was explained [8] by assuming that the motion of one particle creates a vortex around it which, at a later time, pushes forward the particle in the initial direction, thereby effectively favoring the persistence of the velocity autocorrelation. A simulation of hard disks (HD) supported this explanation through the direct visualization of the velocity field of the surrounding particles, which showed a rotary character [8]. In this way, a coupling of the self motion with many-particle dynamics was introduced.

\footnotetext{
*ubaldo.bafile@isc.cnr.it
}

Soon after the Alder and Wainwright discovery, a number of works were devoted to the purpose of putting this finding onto a theoretical base. Papers by various authors [9-11] used hydrodynamic arguments to arrive at a formulation of the LTT behavior in terms of the power law expression $A t^{-n / 2}$ for an $n$-dimensional system. This result was obtained without specific assumptions on the interaction potential, therefore its validity is not limited to the hard-sphere case.

A different kind of approach was followed by exploiting kinetic theory for HS and HD fluids to account for sequences of the so-called correlated ring collisions [12]. The same power law behavior as before was obtained in the limit of vanishing wave vectors where the medium is viewed as a continuum. Moreover, both theories also provide the same expression for the amplitude coefficient $A$ (see below).

The results of the two theories differ in that the former derives the $t^{-n / 2}$ dependence as representing the asymptotic time behavior of the VAF, while in the latter there is no claim of such a property. Actually, in Ref. [12] it is remarked that, if higher-order terms are taken in an iterative calculation of the VAF, time dependencies other than $t^{-n / 2}$ appear at longer times than those that can be usually accessed by MD.

Further simulation studies have concerned model fluids interacting with continuous potentials, in various thermodynamic conditions, aiming at an unambiguous assessment of the LTT [13-20]. In fact, the effect of the mechanisms assumed to lie at the basis of the LTT makes it not observable under all conditions. For example, in too dilute gases the vortex formation may not be sustained, while at high density other dynamical effects, such as backscattering due to the bouncing of atoms off near neighbors, may become dominant and mask the LTT [14].

In summary, a power-law decay has been established for various model fluids in at least a range of their thermodynamical states, although debated through confirmations and refutations, as clearly recalled in Ref. [15]. In particular, a recent two-dimensional HD simulation performed on a very 
large system [19] has shown clear deviations of the VAF from the $t^{-1}$ decay predicted by theory for a 2D system. This result casts some doubt on the validity of the power law as the asymptotic time dependence also in real fluids.

The relevance of the VAF is, however, far from being confined to its very-long-time behavior. The initial decay was also studied, mostly by looking for an appropriate modeling of a memory function to be inserted into a generalized Langevin equation [2,5]. This memory function was assumed to include two parts: one accounting for fast relaxation through binary collisions, the other collectively describing slower decay processes related to sequences of correlated (i.e., nonbinary) collisions. The latter processes display a more collective character than the binary collision mechanism [2] and are expected to eventually merge into the LTT phenomenon.

The relative strengths of the various processes responsible for the VAF decay clearly depend on the thermodynamical state of the fluid, and their change is reflected by the accompanying changes in the shape of the VAF. Thus, one would ultimately aim at a well-founded approach to the VAF analysis covering the whole time range from zero up to the longest time for which a VAF can be determined, including the LTT range if detected, and in the whole fluid-state $(\rho, T)$ space.

In this paper, we pursue this goal by applying a substantially different method, not attempted so far. This is based on a recently presented general theory [21-23] that describes the time dependence of any correlation function of dynamical variables in many-particle Hamiltonian systems as an infinite series of (complex and/or real) exponentials. This theoretical approach, which does not resort to any a priori hypotheses on what dynamical regimes occur in the fluid, also applies naturally to the VAF and evidently opens an interesting issue with respect to the current schemes of interpretation.

We have performed a series of MD simulations of a Lennard-Jones (LJ) fluid, checking for the existence of a $t^{-3 / 2}$ LTT in a limited time range, as reported in Sec. II, and then analyzing the full VAF in terms of the theory just mentioned (Sec. III). The results are presented and discussed in Sec. IV, and the conclusions are summarized in Sec. V.

\section{SIMULATIONS}

We have carried out MD simulations along the slightly supercritical $T^{*}=1.35$ isotherm of the LJ (12-6) fluid. (As usual, variables marked by an asterisk denote reduced quantities made dimensionless by appropriate combinations of the LJ energy and length parameters $\epsilon$ and $\sigma$ and the atomic mass $m$; thus, $T^{*}=k_{\mathrm{B}} T / \epsilon$, with $k_{\mathrm{B}}$ the Boltzmann constant.) The seven simulated densities $\left(\rho^{*}=\rho \sigma^{3}=0.30,0.35,0.40, \ldots, 0.60\right)$, ranging from the critical one to a value about halfway between the critical and the triple point density, were chosen because we expected that such a medium-density gas would exhibit a clearly discernible LTT in the correlation function. In fact, for our lowest density state, a very careful study [15] has already given evidence of a $t^{-3 / 2}$ LTT. Therefore, we have chosen to work with the same sample size of $N=10976$ particles (at all densities) and to use a rather large force cutoff of $r_{c}=6.5 \sigma$, in order to be fully consistent with Ref. [15].

To maintain the system at the required temperature, we have employed a Gaussian thermostat [24]. The results of

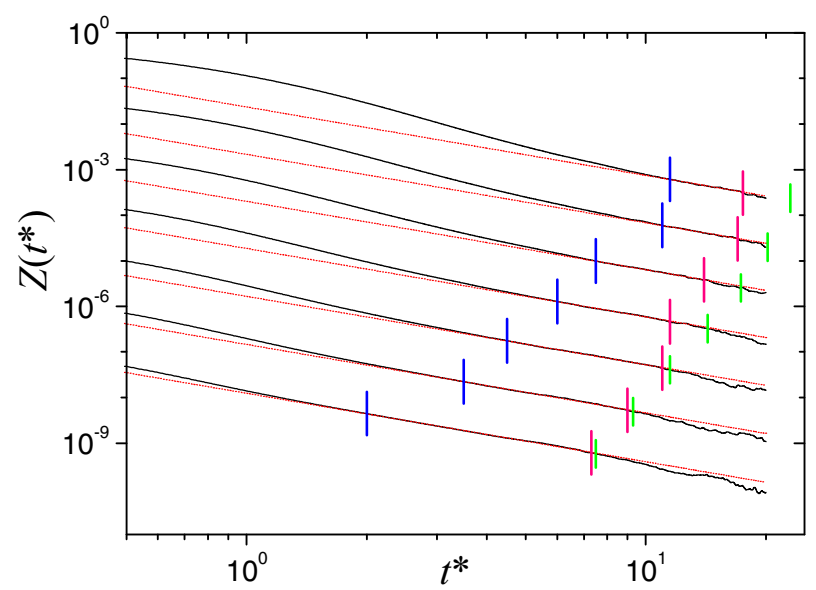

FIG. 1. (Color online) $Z\left(t^{*}\right)$ from simulation (black solid lines) is plotted for $t^{*} \geqslant 0.5$ showing its approach to the $\left(t^{*}\right)^{-3 / 2}$ behavior (red straight lines in the log-log scale). The curves are plotted in order of increasing density from top $\left(\rho^{*}=0.30\right)$ to bottom $\left(\rho^{*}=0.60\right)$, and for clarity each curve is shifted downwards by a factor of 10 with respect to the preceding one. The vertical bars mark the values of $t_{1}^{*}$ (blue), $t_{2}^{*}$ (pink), and $t_{\mathrm{R}}^{*}$ (green). See text for details.

an isokinetic simulation have been checked to be consistent with the correlation function obtained in the microcanonical ensemble. The equations of motion were integrated by means of the simple leapfrog algorithm in the formulation of Brown and Clarke [25] with a timestep of $\Delta t^{*}=0.001$. Here, $t^{*}$ denotes the reduced time $t^{*}=t / \sqrt{m \sigma^{2} / \epsilon}$. To speed up the calculation of the forces, link cells and neighborhood tables were used [26].

At each thermodynamic state we have performed ten independent runs of $10^{7}$ time steps each. We calculated the normalized velocity autocorrelation function

$$
Z(t)=\frac{\langle\mathbf{v}(0) \cdot \mathbf{v}(t)\rangle}{\left\langle\mathbf{v}(0)^{2}\right\rangle},
$$

where $\mathbf{v}(t)$ is the velocity of a particle at time $t$ and $\langle\cdots\rangle$ includes an average over all $N$ particles. $Z(t)$ was computed on a grid with spacing $5 \Delta t^{*}=0.005$ from zero up to a maximum time lag of $t^{*}=20$. The ten individual correlation functions were then averaged to provide, for each $t^{*}$, a mean value of the VAF at that time lag, as well as the standard error of the mean, which could be used as a measure of uncertainty in the subsequent fitting procedures. For all thermodynamic states considered here, $Z(t)$ is shown in Fig. 1 as a function of the reduced time $t^{*}$.

It is well known $[1,26,27]$ that the use of periodic boundary conditions may introduce distortions into correlation functions, depending on the size of the simulation box, for time lags greater than the so-called recurrence time. The latter is usually taken to be the time $t_{\mathrm{R}}$ it takes a density fluctuation to propagate over a distance equal to the box length traveling at the speed of sound $c_{\mathrm{s}}$, i.e., $t_{\mathrm{R}}=(N / \rho)^{1 / 3} / c_{\mathrm{s}}$, for a cubical box. Reduced values of $t_{\mathrm{R}}$, calculated using sound speed data from Refs. [28,29], are reported in Table I. They agree well with values obtained from the determination of $c_{\mathrm{S}}$ in Ref. [30]. We also displayed $t_{\mathrm{R}}$ in Fig. 1, where the recurrence problem 
TABLE I. Reduced recurrence time for the simulations, Enskog mean time between collisions for equivalent hard spheres, and values, in units of $\tau_{\mathrm{E}}$, of the time range limits where the $A t^{-3 / 2}$ behavior is an appropriate representation of the VAF data. In the last two lines the reduced diffusion coefficients from this work and from Ref. [30] are compared.

\begin{tabular}{lccccccc}
\hline \hline$\rho^{*}$ & 0.30 & 0.35 & 0.40 & 0.45 & 0.50 & 0.55 & 0.60 \\
\hline$t_{\mathrm{R}}^{*}$ & 23.0 & 20.2 & 17.3 & 14.3 & 11.5 & 9.3 \\
$\tau_{\mathrm{E}}$ & 0.263 & 0.208 & 0.168 & 0.137 & 0.112 & 0.093 \\
$t_{1} / \tau_{\mathrm{E}}$ & 43 & 53 & 45 & 44 & 40 & 0.077 \\
$t_{2} / \tau_{\mathrm{E}}$ & 67 & 82 & 84 & 84 & 98 & 97 \\
$D^{*}$ (this work) & 0.615 & 0.513 & 0.434 & 0.369 & 0.313 & 0.266 \\
$D^{*}$ (from Ref. [30]) & 0.612 & 0.516 & 0.432 & 0.365 & 0.308 & 0.260 \\
\hline \hline
\end{tabular}

manifests itself in a reduction of the VAF intensity at the end of the reported time range. Actually, the recurrence time $t_{\mathrm{R}}$ slightly overestimates the time where problems with the simulation begin to show up, but this effect tends to diminish with increasing density.

We performed a two-parameter fit of the function $A t^{-p}$ to the tail of $Z(t)$ data in variable time windows, and for each density we determined empirically the widest interval $t_{1} \leqslant t \leqslant$ $t_{2}$ within which the values of $A, p$, and the reduced $\chi^{2}$ were constant. The values of $t_{1}$ and $t_{2}$, also shown in Fig. 1, appear consistent with what one would choose by visual inspection. The fitted $p$ was fluctuating around an average of $1.500 \pm$ 0.005 without any density trend. Then, a new fit with $p$ fixed at $3 / 2$ gave, at each density, a best choice for $A$. We used these values in Fig. 1 to draw the power-law dependence.

In Fig. 2 we compare the fitted values of $A$ with the theoretical predictions for the LTT. As said, all theories already quoted [10-12] provide the same expression, given by [2]

$$
A=\frac{1}{12 \rho[\pi(D+v)]^{3 / 2}},
$$

where $D$ is the self-diffusion coefficient, and $v=\eta /(m \rho)$ and $\eta$ are the kinematic and shear viscosities, respectively. We

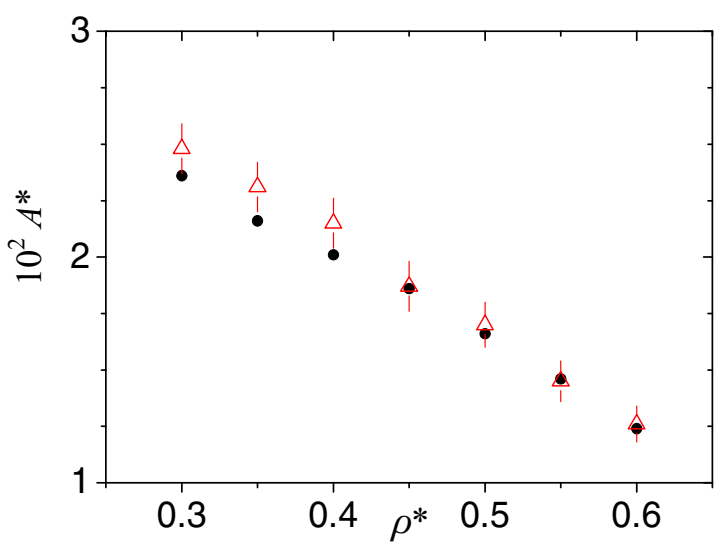

FIG. 2. (Color online) Amplitude $A^{*}$ of the power-law behavior. The theoretical values (red triangles), calculated via Eq. (2), are shown together with those obtained from fitting the function $A t^{-3 / 2}$ to the VAF data in the range $t_{1} \leqslant t \leqslant t_{2}$ (see text). Error bars for the theoretical values are estimated from the uncertainties in the quantities $D$ and $v$. The errors on the fitted points are within the symbol size. estimated $D$ from the time integral of the VAF [1,2], obtaining the values reported in Table I, where they can be compared with those of Ref. [30]. Taking also $\eta$ from Ref. [16], we used Eq. (2) to calculate $A$ in good agreement with the fitted amplitude, especially at the higher densities. The slight discrepancy at lower densities might be due to the fact that the fit range $t_{1} \leqslant t \leqslant t_{2}$ shrinks with decreasing density.

Characteristic times of the various processes involved will be measured in units of "collision times," since all dynamical effects are ultimately determined by repeated collisions between particles, which become more frequent the higher the density. As a reference collision time it is customary to take the Enskog mean free time $\tau_{\mathrm{E}}$ of a corresponding HS fluid, the only system where such a quantity can be rigorously defined. For hard spheres of diameter $\sigma$, the Enskog kinetic theory predicts a mean time between collisions given by [5]

$$
\tau_{\mathrm{E}}=\frac{1}{4 \rho \sigma^{2} g(\sigma)} \sqrt{\frac{m}{\pi k_{\mathrm{B}} T}} .
$$

Here an approximate expression for the contact value of the pair distribution function $g(r)$ may be obtained from the Carnahan-Starling equation of state for hard spheres [1]:

$$
g(\sigma)=\frac{1-\pi \rho \sigma^{3} / 12}{\left(1-\pi \rho \sigma^{3} / 6\right)^{3}} .
$$

We have inserted the $\mathrm{LJ}$ densities into the above formulas to get the values for $\tau_{\mathrm{E}}$ listed in Table I. In the same table is also shown that the ratios $t_{1} / \tau_{\mathrm{E}}$ and $t_{2} / \tau_{\mathrm{E}}$ are only weakly dependent on density and correspond, respectively, to 30-50 and 70-100 collisions, confirming the multicollisional origin of the LTT.

\section{MULTIEXPONENTIAL ANALYSIS}

While in the previous Section we have confirmed that the $t^{-3 / 2}$ law is a good LTT representation in the cases studied here, the goal of this work is to obtain a full account of the whole VAF time dependence starting at $t=0$, since no such result is available yet.

We follow the theoretical approach recently presented [21-23], which states that the generalized Langevin equation for a normalized autocorrelation function $C(t)$ of a classical many-body system has an exact solution written as an infinite sum of exponential functions (we need to consider positive $t$ 
only, as $C(t)$ is an even function), i.e.,

$$
C(t)=\sum_{j=1}^{\infty} I_{j} \exp \left(z_{j} t\right),
$$

where $I_{j}$ and $z_{j}$ are mode amplitudes and frequencies, respectively, in an inherent eigenmode decomposition. Such modes can be associated to relaxation channels in the system. If $I_{j}$ and $z_{j}$ are complex quantities, the corresponding mode and its complex conjugate are both present in the series and, taken together, they represent an exponentially damped oscillation. Otherwise, real $I_{j}$ and $z_{j}$ define a purely exponential decay. In all cases, the real part of $z_{j}$ is negative ensuring the decay to zero of $C(t)$ in the $t \rightarrow \infty$ limit. The zero-time properties of $C(t)$ and the frequency moments of the spectrum $\hat{C}(\omega)$, obtained by Fourier transformation of Eq. (5), are linked by the relationship

$$
\left(\frac{d^{k} C(t)}{d t^{k}}\right)_{t=0}=i^{k}\left\langle\omega^{k}\right\rangle \equiv i^{k} \int_{-\infty}^{\infty} d \omega \omega^{k} \hat{C}(\omega) .
$$

Since in a system of classical particles interacting with a continuous potential any nonpathological correlation function is even in time and infinitely often differentiable at $t=0$, Eq. (6) implies that $\left\langle\omega^{k}\right\rangle$ is zero for all odd $k$ and has finite values for all even $k$, and also that $I_{j}$ and $z_{j}$ satisfy, for $k \geqslant 0$, a set of sum rules of the form

$$
\sum_{j=1}^{\infty} I_{j} z_{j}^{k}=i^{k}\left\langle\omega^{k}\right\rangle .
$$

Specific models for the autocorrelation $C(t)$ of a dynamical variable are obtained as approximations of Eq. (5) through truncation of the infinite series. In Ref. [21] we have shown that the approximation involved is related to the accuracy of the description of the time evolution of the dynamical variable. When a finite number of exponential terms is retained, Eqs. (6) and (7) can only be valid for $k$ up to a certain value depending on the approximation level and the model assumed.

Thus, besides being the rigorous solution to the evolution of $C(t)$, Eq. (5) has also the merit of permitting the explicit control of the approximation involved in setting up a specific model for $C(t)$. This is done through a convenient choice of the number of exponential terms to be retained, depending on the extension and accuracy of available data, and on the need to avoid a statistically meaningless overparametrization of the fitting function. Moreover, apart from the trivial normalization condition $\sum_{j} I_{j}=1$ (obtained from Eq. (5) for $t=0$ ), the fit parameters can also be constrained to obey a certain number of sum rules $\sum_{j} I_{j} z_{j}^{k}=0$ for odd $k$, each of them ensuring the finiteness of the spectral moment $\left\langle\omega^{k+1}\right\rangle$ [31].

In the whole range $0 \leqslant t \leqslant t_{2}$ of our reliable LJ data, a remarkably accurate description of the VAF is obtained with two pairs of complex conjugate exponentials and four real exponentials. We have checked that, at all thermodynamical states investigated, this set of exponential terms is the smallest one to be kept in Eq. (5) in order to provide the required accuracy of the fit, which means that, if one wants to avoid an unjustified overparametrization of the fit function, this is univocally determined.
We have specified four constraints provided by the sum rules Eq. (7) with $k=0,1,3,5$. From the computational point of view, the fitting of the multiexponential model described in the text is performed by means of a program run in the Matlab [32] environment, where the core least-squares-fit calculation is carried out by a built-in Levenberg-Marquardttype minimization routine. Weights defined via the statistical uncertainties evaluated in the VAF computation are used for the definition of the $\chi^{2}$ to be minimized. The implementation of the sum rule constraints is performed by applying linear algebra routines to the solution of the system of equations that give the nonfitted parameters in terms of the fitted ones.

Imposing the constraints indicated before does not merely provide a useful reduction in the number of free-fit parameters, but also renders the model used here a much more accurate description of the VAF and its spectrum than it is customarily obtained. We are not aware, in fact, of any study of dynamical spectra where the finiteness of the sixth frequency moment was imposed (through the sum rule Eq. (7) with $k=5$ ).

The reason why we do not enforce any sum rules with even $k$ (apart from the normalization condition) is that the corresponding zero-time derivatives of the autocorrelation function are, in general, unknown. In the case of the VAF, for example, the computation of the fourth derivative of $Z(t)$ at $t=0$ requires the knowledge of the three-body distribution function $g_{3}\left(\mathbf{r}_{12}, \mathbf{r}_{13}\right)$, and higher-order derivatives have even more involved expressions [33]. However, for the second derivative of $Z(t)$, the theoretical expression at $t=0$ is easy to calculate and can be compared to the result obtained from the fit in order to provide a consistency check of our analysis, as done in the next section.

\section{RESULTS AND DISCUSSION}

A first check of the consistency of the applied fit function is its fulfillment of the $k=2$ sum rule. The VAF has the well-known short time expansion in even powers of $t$ [2,5],

$$
Z(t)=1-\Omega_{0}^{2} \frac{t^{2}}{2}+\ldots,
$$

where

$$
\Omega_{0}^{2}=\frac{\rho}{3 m} \int d \boldsymbol{r} \nabla^{2} \phi(r) g(r) .
$$

Here $\Omega_{0}$ is the so-called Einstein frequency, and $\phi(r)$ is the interaction potential. For the $\mathrm{LJ}$ case this reads

$$
\Omega_{0}^{2}=\frac{32 \pi \rho \epsilon}{m} \int_{0}^{\infty} d r g(r)\left[22\left(\frac{\sigma}{r}\right)^{12}-5\left(\frac{\sigma}{r}\right)^{6}\right],
$$

which we calculated using the results of $g(r)$ simulations at the various densities. On the other hand, by combining Eqs. (7) and (6), one obtains

$$
\Omega_{0}^{2}=\sum_{j=1}^{\infty} I_{j} z_{j}^{2}
$$

Putting in this sum the finite number of terms used in our fit function, $\Omega_{0}$ can be evaluated from the fitted $I_{j}$ and $z_{j}$. The two values of $\Omega_{0}^{2}$ obtained from Eqs. (10) and (11) agree within $2 \%$ at $\rho^{*}=0.30$ and better than $1 \%$ at all higher densities. 

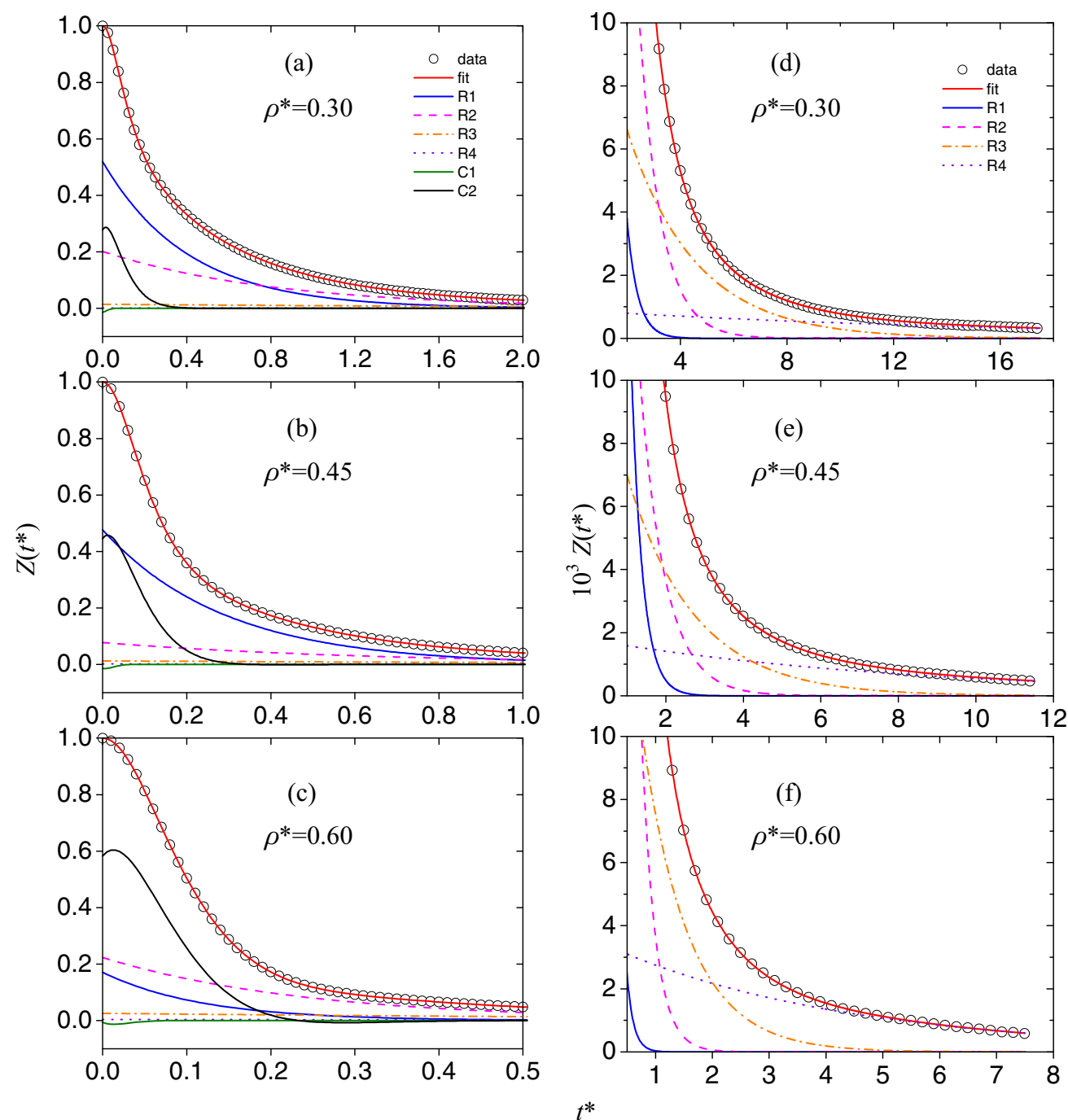

FIG. 3. (Color online) The simulated $Z\left(t^{*}\right)$ is displayed (symbols) for the three indicated densities together with the multiexponential fit described in the text (red solid line through the data points). In the left frames [(a)-(c)], referring to short times, the various components of the fit function are also displayed separately according to the legend given in the top panel. R1 to R4 denote the real exponential terms ordered by increasing decay time. $\mathrm{C} 1$ is the sum of the two complex conjugate exponentials of the first pair, amounting to a damped oscillatory function. $\mathrm{C} 2$ is the analogous quantity for the second pair of complex terms. R3, R4, and C1 are hardly visible on the scale of the figures. In the right panels [(d)-(f)], covering the remaining time range up to $t_{2}^{*}$, only the real terms are shown while $\mathrm{C} 1$ and $\mathrm{C} 2$ have already decayed to zero. For graphical clarity, not all available data points have been displayed.

Figure 3 shows that the multiexponential fit curves agree perfectly with the VAF data at all densities. In particular, for times between $t_{1}$ and $t_{2}$ the fit residuals are at least as small as in a fit with the power law. The various components of the fit model are also shown in Fig. 3 for a more detailed understanding of the results.

The oscillatory modes (labeled $\mathrm{C} 1$ and $\mathrm{C} 2$ ) are strongly damped and one of them $(\mathrm{C} 1)$ is characterized by a very low intensity. These modes and the two faster decaying real exponentials (R1 and R2) mainly determine the VAF behavior at short and intermediate times, respectively. The role of the slower decaying exponentials (R3 and R4) can instead be better appreciated at the larger times displayed in the right panels of the figure. Notably, the LTT range $\left(t \geqslant t_{1}\right)$ is described by the R3 and, predominantly, R4 terms. The latter, however, is apparently characterized by a time constant much longer than the other modes, suggesting the onset of a different (hydrodynamic-like) regime for the fluid. With increasing density, the short-time oscillatory mode progressively grows in importance with respect to the two fast decaying real modes R1 and R2. This is suggestive of the onset of bouncing effects in cages surrounding a given atom, which are typical of a dense liquid but may here be already beginning to form as the packing fraction grows. Moreover, the intensity of R4 also increases with density, in agreement with an earlier occurrence of hydrodynamic conditions and with the greater values of $Z\left(t_{1}\right)$ at higher densities, which shows the growing importance of the LTT contribution to the VAF and its integral, which is connected to the diffusion constant.

The fact that two exponential terms (R3 and R4) provide an accurate representation of the MD data for $t_{1} \leqslant t \leqslant t_{2}$, i.e., in the same range where the algebraic $t^{-3 / 2}$ dependence 
is detected, should not appear as a contradiction. Of course, this is not an absurd claim that the sum of two exponentials amounts to an algebraic function, but simply the statement that the two representations can only be assessed in the comparison with data of a given finite precision. In this respect, we already noted that in the above quoted range the multiexponential fit is at least as good as the $t^{-3 / 2}$ one.

It is intrinsic to the theory that the VAF decays by superposition of effects that can be identified with relaxation channels of different nature. Here we learn, however, that these channels are limited in number to a few, and still fewer of them are sufficient to account for the LTT. Moreover, it is a natural consequence of the theory that, if VAF data were obtainable in a significantly wider range, additional slower-decay modes should eventually be added to the model and that these would account for contributions to the VAF of lesser and lesser intensity and located in the very far tail.

Turning to the quantitative analysis of fit results, we write $z_{j}=-1 / \tau_{j}$ or $z_{j}=\left(-1 / \tau_{j}\right)+\mathrm{i} \omega_{j}$ for a real or complex mode, respectively, and, by considering the scaled quantities $\tau_{j} / \tau_{\mathrm{E}}$ for the time decays and $\omega_{j} \tau_{\mathrm{E}}$ for the complex mode oscillation frequencies, we are able to estimate the number of collisions involved in the loss of correlation brought about by the various processes related to each fitted mode in Eq. (5). Figure 4(a) shows the density dependence of $\tau / \tau_{\mathrm{E}}$. The oscillatory modes have the shortest decay times, which, together with that of R1, are less than, or of the order

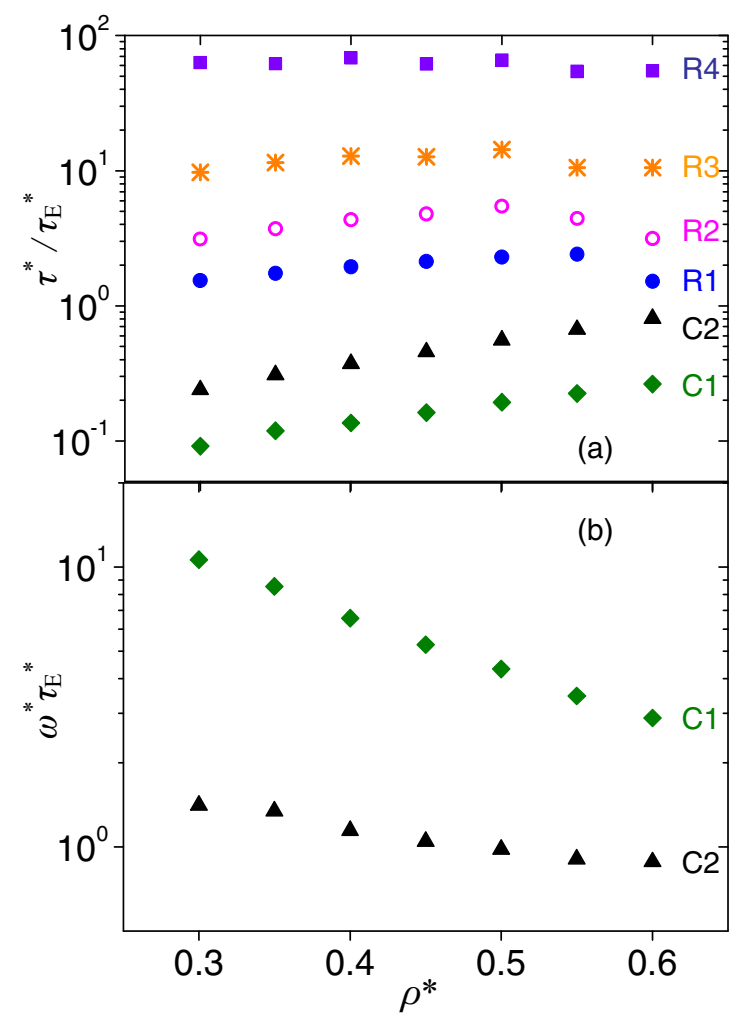

FIG. 4. (Color online) Parameters of the exponential fit. (a) Decay times $\tau_{j}$ of the fitted exponentials in units of $\tau_{\mathrm{E}}$ as functions of density. The various modes are identified by labels as described in the caption of Fig. 3. (b) The product $\omega_{j} \tau_{\mathrm{E}}$ is shown for the two complex modes. of, one collision time. R2 is also a relatively fast process related to a very small number of collisions. These modes correspond qualitatively to the mentioned binary collision components. There also appears a mode (R3) with $\tau / \tau_{\mathrm{E}}$ of the order of ten. This intermediate value denotes a process where more collisions are involved but still not in such a number as to suggest a truly cooperative damping mechanism. However, the most striking result is the great gap between the first three real modes and the fourth one (R4), which has a decay time of the order of 60 collisions, revealing its true multiparticle, collective origin. These observations are quantitative confirmations of the Alder and Wainwright findings, but also show that the loss of velocity correlation is a consequence of more than one relaxation process, ranging from the single-collision fast processes $\mathrm{R} 1, \mathrm{C} 1$, and $\mathrm{C} 2$ to the slow, hydrodynamic-like one, R4.

A clear difference is also evident in the density dependence, which is appreciable for the oscillatory modes but hardly detectable for the decaying ones on the logarithmic scale of Fig. 4(a). This can be interpreted by noting that the exponential decays R1 to R4 already offer a range of values broad enough to account for possible density effects; on the contrary, the oscillatory components are only two and need to adapt themselves to the change of damping condition brought about by a growing density.

Figure 4(b) shows that the oscillation frequencies of the complex modes confirm both their fast nature and their greater sensitivity to density changes. It is worthwhile to note that all parameters plotted in Fig. 4 display a smooth dependence on density.

As already visible in Fig. 3, the slowly decaying real exponentials R3 and R4 have very small intensities. However, they account for the total VAF in the very important long-time range where all other terms have already fully decayed to zero. In Fig. 5, which displays the mode intensities as functions of density, it is seen that (i) C2 and R1 are the most intense

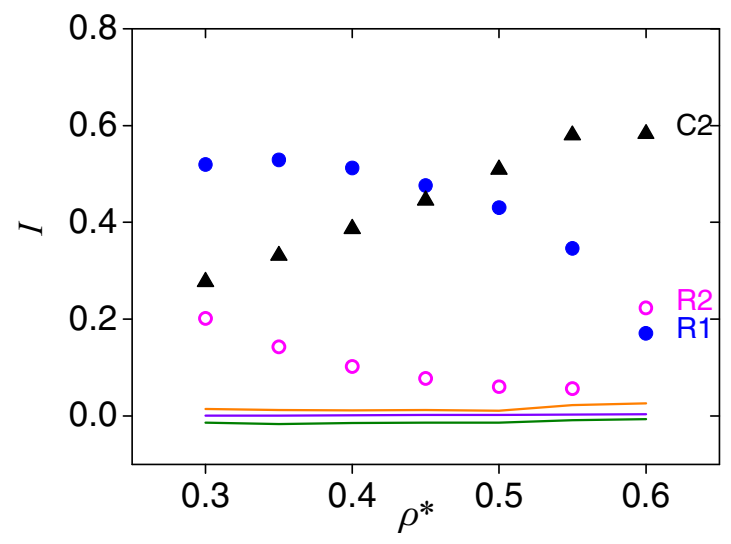

FIG. 5. (Color online) Amplitudes $I$ of the fitted modes as functions of density. The three most intense modes are shown as symbols and identified by the same labels as in Fig. 4. The other modes have amplitudes too small to be clearly distinguishable on the scale of the figure and are displayed as continuous lines through the points, for graphical reasons. For the complex modes the amplitudes of the sums of each pair of complex conjugate terms, given by $2 \operatorname{Re} I$, are shown. 
TABLE II. Percent contribution, of the fitted modes grouped as shown in the first column, to the integral of the VAF. The numbers in each column add up to 100 percent.

\begin{tabular}{lrcccccc}
\hline \hline$\rho^{*}$ & 0.30 & 0.35 & 0.40 & 0.45 & 0.50 & 0.55 & 0.60 \\
\hline $\mathrm{C} 1+\mathrm{C} 2$ & 7.81 & 10.64 & 14.28 & 18.56 & 23.63 & 30.12 & 34.14 \\
$\mathrm{R} 1+\mathrm{R} 2$ & 81.04 & 78.09 & 73.85 & 68.22 & 62.93 & 50.77 & 44.49 \\
$\mathrm{R} 3+\mathrm{R} 4$ & 11.14 & 11.27 & 11.87 & 13.21 & 13.44 & 19.11 & 21.38 \\
\hline \hline
\end{tabular}

modes, followed by R2, but (ii) the density dependence clearly suggests that the oscillating mode gains intensity at the expenses of the fastest decaying real exponential one.

More insight into the effects of increasing density is gained by considering the relative weights $w_{j}=\left(I_{j} / z_{j}\right) / \sum_{h}\left(I_{h} / z_{h}\right)$ of the various modes defined as their fractional contribution to the integral of the VAF. In order to present the density trends in a simpler but more effective way, in Table II we combined the modes into three groups consisting of the complex modes, the two faster real ones, and the two slower ones. The interpretation of Table II is straightforward. By moving toward dense fluid conditions, the slow modes play an increasing role to account for the hydrodynamic-like behavior, which is also responsible for the emergence of the LTT. On the other hand, for the fast modes $(\mathrm{C} 1+\mathrm{C} 2$ and $\mathrm{R} 1+\mathrm{R} 2)$, essentially governed by single-collision processes, one observes a shift toward a progressively stronger oscillatory nature in parallel with the formation of better structured nearest-neighbor cages. This is the same phenomenon that, when the density of the liquid state is reached, will have increased to the point of making the total VAF display a negative part.

\section{CONCLUSION}

We have shown that the multiexponential representation of correlation functions applies perfectly, with only a small number of terms, to the description, up to very large times, of the VAF of a supercritical LJ fluid. A collisional analysis, together with its density evolution, allowed us to distinguish the nature of the mechanisms contributing to diffusion and the ultimate loss of velocity correlation. The long-discussed concepts of "binary" (i.e., fast) and "nonbinary" (i.e., slower) processes intervening in the VAF time dependence are found to correspond directly to decay channels whose intensities and lifetimes can be measured. Moreover, when these modes possess an oscillatory character, their frequencies are also obtained from the analysis in a natural way.

The presence of a very slow process, with a decay time much longer than that of the other modes and measured on the scale of several tens of collision times, shows that the analysis here performed is consistent with the originally proposed interpretation of the LLT, but, at the same time, provides a unified description of all dynamical properties reflected in the time evolution of the single-atom velocity correlation.

The application of the theoretical treatment of Refs. [21-23] to the VAF case allows for a method of interpreting $Z(t)$ data quite different and much more farreaching than the one limiting the analysis to the assessment of an LTT. However, given the generality of the theoretical results as far as their field of application is concerned, the work presented here can also be considered as a meaningful illustration of a method of analysis that can be extended to a variety of time correlation and spectral data of interest in many fields of matter physics.

For example, from the expression of a correlation function as a series of exponential functions, it follows that the corresponding Green-Kubo integral also gives a transport coefficient in the form of a series. Besides being a nice result "per se," this means that, once the parameters of the exponentials are fitted to good data, the calculation of the corresponding transport coefficient is also obtained. A similar approach is exploited in Ref. [34], where extrapolation to infinite time of the Green-Kubo integrand is performed by means of a fitted function empirically modeled as a double exponential. Our method could be applied to put these analyses on a rigorous base.

\section{ACKNOWLEDGMENT}

We thank Walter Penits for the maintenance of, and his kind help with, the HTCondor system [35] and Daniele Colognesi for the computation of the LJ pair distribution functions.
[1] J. P. Hansen and I. R. McDonald, Theory of Simple Liquids (Academic Press, London, 1986).

[2] U. Balucani and M. Zoppi, Dynamics of the Liquid State (Clarendon, Oxford, 1994).

[3] T. Gaskell and S. Miller, J. Phys. C: Solid State Phys. 11, 3749 (1978).

[4] T. Gaskell and S. Miller, J. Phys. C: Solid State Phys. 11, 4839 (1978).

[5] J. P. Boon and S. Yip, Molecular Hydrodynamics (Dover, New York, 1980).

[6] D. Levesque and L. Verlet, Phys. Rev. A 2, 2514 (1970).

[7] B. J. Alder and T. E. Wainwright, Phys. Rev. Lett. 18, 988 (1967).

[8] B. J. Alder and T. E. Wainwright, Phys. Rev. A 1, 18 (1970).

[9] K. Kawasaki, Phys. Lett. 32A, 379 (1970).
[10] M. H. Ernst, E. H. Hauge, and J. M. J. van Leeuwen, Phys. Rev. Lett. 25, 1254 (1970).

[11] M. H. Ernst, E. H. Hauge, and J. M. J. van Leeuwen, Phys. Rev. A 4, 2055 (1971).

[12] J. R. Dorfman and E. G. D. Cohen, Phys. Rev. A 6, 776 (1972).

[13] D. Levesque and W. T. Ashurst, Phys. Rev. Lett. 33, 277 (1974).

[14] A. McDonough, S. P. Russo, and I. K. Snook, Phys. Rev. E 63, 026109 (2001).

[15] R. F. A. Dib, F. Ould-Kaddour, and D. Levesque, Phys. Rev. E 74, 011202 (2006).

[16] K. Meier, A. Laesecke, and S. Kabelac, J. Chem. Phys. 121, 3671 (2004).

[17] K. Meier, A. Laesecke, and S. Kabelac, J. Chem. Phys. 121, 9526 (2004). 
[18] S. R. Williams, G. Bryant, I. K. Snook, and W. van Megen, Phys. Rev. Lett. 96, 087801 (2006).

[19] M. Isobe, Phys. Rev. E 77, 021201 (2008).

[20] R. E. Ryltsev and N. M. Chtchelkatchev, J. Chem. Phys. 141, 124509 (2014).

[21] F. Barocchi, U. Bafile, and M. Sampoli, Phys. Rev. E 85, 022102 (2012).

[22] F. Barocchi and U. Bafile, Phys. Rev. E 87, 062133 (2013).

[23] F. Barocchi, E. Guarini, and U. Bafile, Phys. Rev. E 90, 032106 (2014).

[24] W. G. Hoover, Computational Statistical Mechanics (Elsevier, Amsterdam, 1991).

[25] D. Brown and J. H. R. Clarke, Mol. Phys. 51, 1243 (1984).

[26] M. P. Allen and D. J. Tildesley, Computer Simulation of Liquids (Clarendon Press, Oxford, 1987).

[27] J. J. Erpenbeck and W. W. Wood, Phys. Rev. A 26, 1648 (1982).
[28] J. O. Hirschfelder, C. F. Curtiss, and R. B. Bird, Molecular Theory of Gases and Liquids (John Wiley \& Sons, New York, 1954).

[29] J. K. Johnson, J. A. Zollweg, and K. E. Gubbins, Mol. Phys. 78, 591 (1993).

[30] K. Meier, Ph.D. thesis, University of the Federal Armed Forces Hamburg, 2002.

[31] U. Bafile, E. Guarini, and F. Barocchi, Phys. Rev. E 73, 061203 (2006).

[32] MATLAB version 7.6 (R2008a), (The MathWorks Inc., Natick, MA, 2008).

[33] B. R. A. Nijboer and A. Rahman, Physica 32, 415 (1966).

[34] Y. Zhang, A. Ohtani, and E. J. Maginn, J. Chem. Theor. Comput. 11, 3537 (2015).

[35] http://research.cs.wisc.edu/htcondor/. 